\title{
Tuberculosis hepática aislada mimetizando un tumor hepático en un paciente en diálisis
}

\author{
Camilo Andrés García-Prada' ${ }^{1}$, Tomás Rodríguez-Yanez', Angie Córdoba-Caballero², Fernando De-La-Vega-Del-Risco³ \\ 'Servicio de Medicina Interna. Universidad de Cartagena. Cartagena. Colombia \\ ${ }^{2}$ Servicio de Medicina General. Universidad del Magdalena. Santa Marta. Colombia \\ ${ }^{3}$ Servicio de Infectología. Hospital Universitario del Caribe. Universidad de Cartagena. Cartagena. Colombia
}

Recibido: 15/06/2020

Aceptado: 16/02/2021

En línea: 30/04/2021

Citar como: García-Prada CA, Rodríguez-Yanez T, Córdoba-Caballero A, De-La-Vega-Del-Risco F. Tuberculosis hepática aislada mimetizando un tumor hepático en un paciente en diálisis. Rev Esp Casos Clin Med Intern (RECCMI). 2021 (abril); 1(6): 32-35. doi: 10.32818/reccmi.a6n1a10.

Cite this as: García-Prada CA, Rodríguez-Yanez T, Córdoba-Caballero A, De-La-Vega-Del-Risco F. Isolated liver tuberculosis mimicking a liver tumor in a patient on dialysis. Rev Esp Casos Clin Med Intern (RECCMI). 2021 (April); 1(6): 32-35. doi: 10.32818/reccmi.a6n1a10.

Autor para correspondencia: Camilo Andrés García-Prada. cgarciap4@unicartagena.edu.co

\section{Palabras clave}

$\triangleright$ Uremia

$\triangleright$ Tuberculosis hepática

$\triangleright$ Hemodiálisis

\section{Resumen}

La tuberculosis (TB) hepática aislada es una forma rara de TB extrapulmonar, incluso en países donde la tuberculosis se comporta como un problema de salud pública. Su diagnóstico es complejo dada la baja especificidad del cuadro clínico y al limitado rendimiento diagnóstico de los estudios microbiológicos para establecer la presencia del Mycobacterium tuberculosis.

Presentamos el caso de un varón de 65 años con enfermedad renal crónica grado 5 en terapia de reemplazo renal con hemodiálisis quien presentó cuadro clínico de 3 días de evolución asociado a dolor abdominal y anorexia. En la ecografía se evidenció lesiones hepáticas hipoecoicas, corroboradas por tomografía de abdomen con hallazgos sugestivos de neoplasia hepática secundaria sin establecerse un origen primario. La biopsia de hígado finalmente condujo al diagnóstico de tuberculosis hepática sin encontrarse compromiso pulmonar o extrapulmonar adicional. Concluimos que las formas extrapulmonares de TB son entidades clínicas que pueden estar precipitadas por condiciones ligadas a inmunosupresión como es la enfermedad renal crónica (ERC).

\begin{abstract}
Isolated tuberculosis (TB) of the liver is a rare form of extrapulmonary presentation of the disease, even in countries where tuberculosis is a public health concern. Given its low incidence, diagnosis is complex since the specificity of the clinical picture and microbiological studies have limited detection of Mycobacterium tuberculosis.

We present the case of a 65 years-old male with grade 5 chronic kidney disease in renal replacement therapy with hemodialysis and a clinical course of 3 days of abdominal pain and anorexia. Imaging studies showed hypoechoic liver lesions on ultrasound, corroborated by simple and contrasted abdominal tomography, in which findings were suggestive of secondary hepatic neoplasia without establishing a primary origin. Finally, a liver biopsy was performed and led to liver tuberculosis diagnosis without additional pulmonary or extrapulmonary involvement. We concluded that extrapulmonary forms of TB are clinical entities that may be precipitated by conditions linked to immunosuppression, such as chronic kidney disease (CKD), particularly in the last stages.
\end{abstract}

Puntos destacados

$\triangleright$ La enfermedad renal crónica es un estado de inmunosupresión reconocido y los síntomas inespecíficos en estos pacientes se deben estudiar en profundidad.

$\triangleright$ La tuberculosis hepática primaria es una presentación rara de la TB extrapulmonar pero se ha asociado a los pacientes en diálisis.

\section{Introducción}

La tuberculosis (TB) sigue siendo un problema importante de salud pública a nivel global, siendo la segunda causa de muerte por enfermedades infecciosas. Se estima que alrededor del 30\% de la población mundial está infectada con M. tuberculosis ${ }^{1}$ y, según la Organización Mundial de la Salud (OMS), en 2016, 
10,4 millones de personas enfermaron de tuberculosis y 1,7 millones murieron La afectación hepática supone menos del 1\% de todos los casos de TB2 , y se asocia generalmente a focos de infección, ya sea pulmonar o en tracto gastrointestinal, o con un estado de inmunosupresión³.

Los casos de tuberculosis hepática aislada o primaria son raros. En la literatura revisada, se informan casos aislados de TB hepática en pacientes en diálisis A continuación, presentamos un caso inusual de TB hepática aislada que imita un tumor en el hígado en un paciente en diálisis.

\section{Caso clínico}

\section{Antecedentes personales}

Varón de 65 años, con antecedentes de hipertensión arterial, diabetes mellitus tipo 2 y enfermedad renal crónica grado 5 en diálisis. Consulta por presentar cuadro clínico de tres de días de evolución caracterizado por: dolor abdominal difuso, tipo cólico, de moderada intensidad; asociado a malestar general, hiporexia, y episodios eméticos de contenido alimenticio.

\section{Exploración física}

Al examen físico se encontraba afebril, con signos vitales en rangos normales, abdomen depresible, doloroso a la palpación de hipocondrio derecho; signo de Murphy negativo, sin signos de irritación peritoneal, y no se halló a la palpación la presencia de masas o vísceromegalias.

\section{Pruebas complementarias y evolución}

En la analítica se reporta anemia moderada normocitica normocromica; leucocitosis con desviación a la izquierda (17.240 células $/ \mathrm{mm}^{3}$ ); con elevación de azoados propio de su enfermedad de base.

Se le realizó una ecografía abdominal en la que se observó imagen hipoecoica, redondeada, de $18 \mathrm{~mm}$ en segmento II hepático; además de imágenes hipoecoicas de 1,6 y 2 cm compatibles con adenomegalias en región de ligamento gastrohepático.

Teniendo en cuenta los hallazgos descritos se solicitó una tomografía axial computarizada (TAC) abdominal con contraste, en la cual se identificó una formación hipodensa, sólida, localizada en el lóbulo izquierdo del hígado segmento II de bordes mal definidos de probable etiología neoplásica (Figuras 1 y 2).

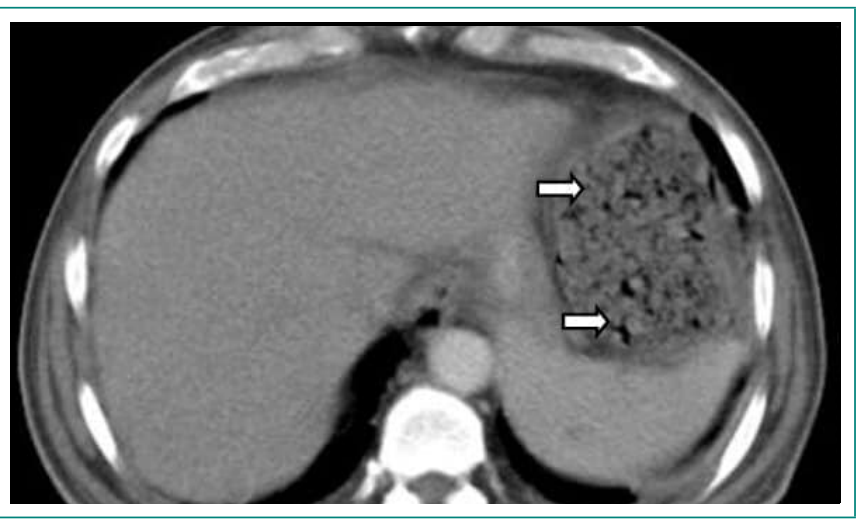

Figura 1. TAC de abdomen con y sin contraste: se evidencia formación hipodensa de $73,47 \times 88,58 \mathrm{~mm}$ de bordes mal definidos en segmento II de lóbulo izquierdo hepático.

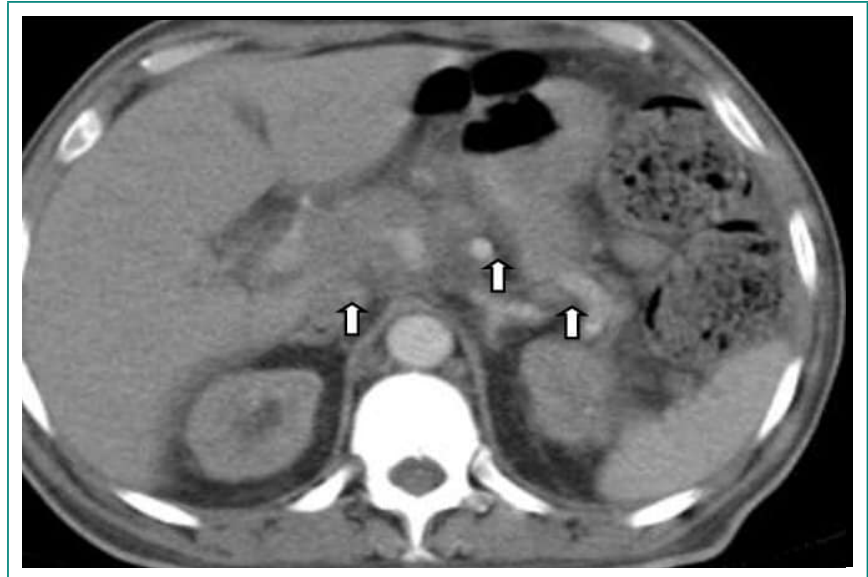

Figura 2. TAC: de abdomen con y sin contraste: se evidencia presencia de pequeños ganglios en el retroperitoneo a la altura de la mesentérica superior y tronco celiaco así como adenomegalias lateroaorticas.

Basados en los hallazgos tomográficos, se realizó biopsia de la lesión de hígado con la sospecha de neoplasia hepática de origen secundario. Sin embargo, el estudio histopatológico mostró un parénquima hepático comprometido por proceso inflamatorio crónico granulomatoso necrotizante (Figuras 3, 4 y 5).

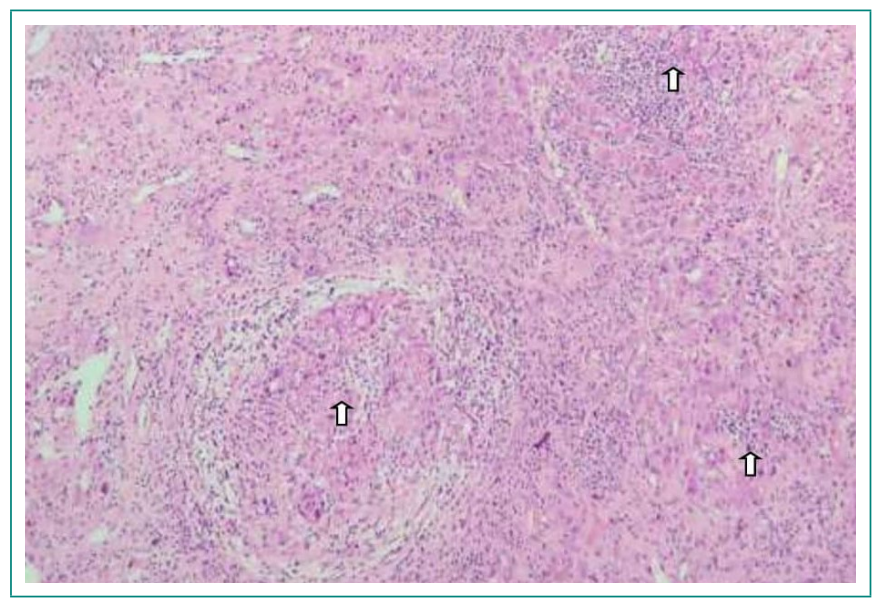

Figura 3. Se observa formación de granulomas necrotizantes asociados a focos de necrosis confluyente e infiltrado linfocitico y formación de agregados linfoides primarios.

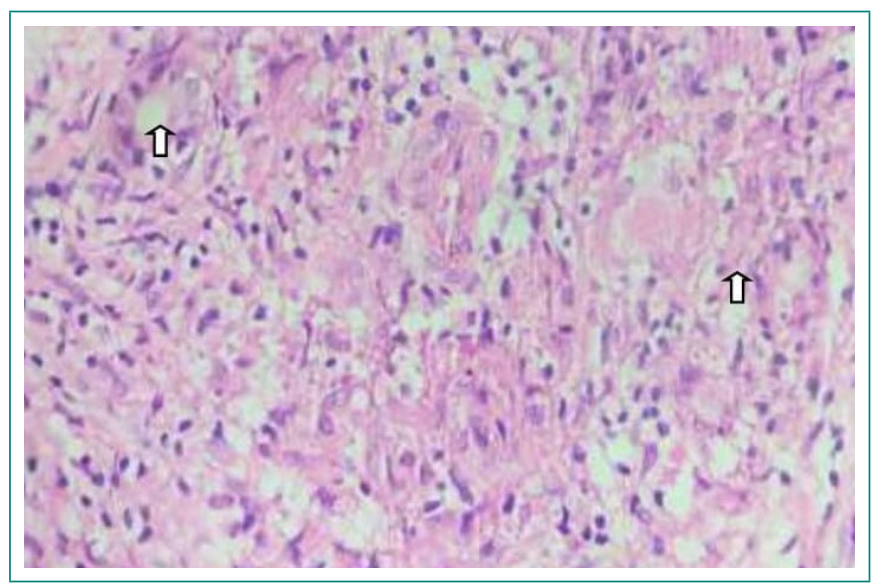

Figura 4. Se observa formación de granulomas necrotizantes con células gigantes multinucleadas tipo Langhans. 


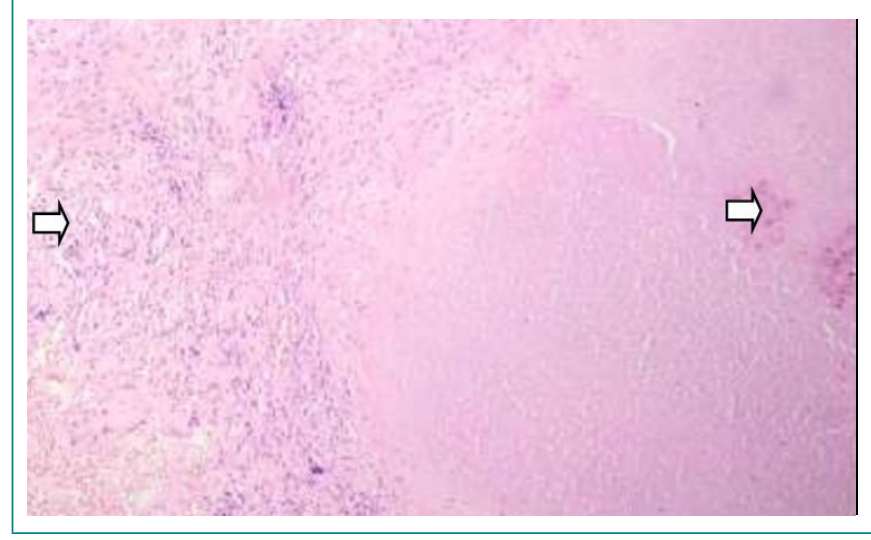

Figura 5. Se identifica fibrosis en banda con nódulos de regeneración y destrucción de ductos biliares.

Teniendo en cuenta la biopsia, datos epidemiológicos y la ausencia de antecedentes o sintomatología clínica asociada a sarcoidosis, se consideró como diagnóstico principal tuberculosis extrapulmonar con compromiso hepático siendo este apoyado por la identificación de BAAR en la coloración de ZiehlNeelsen de la muestra.

Adicionalmente, se solicitó radiografía de tórax (Figura 6) en la cual no se encontró hallazgos sugestivos de tuberculosis pulmonar; además de baciloscopia seriada, los cuales arrojaron resultados negativos; descartándose así tuberculosis pulmonar.

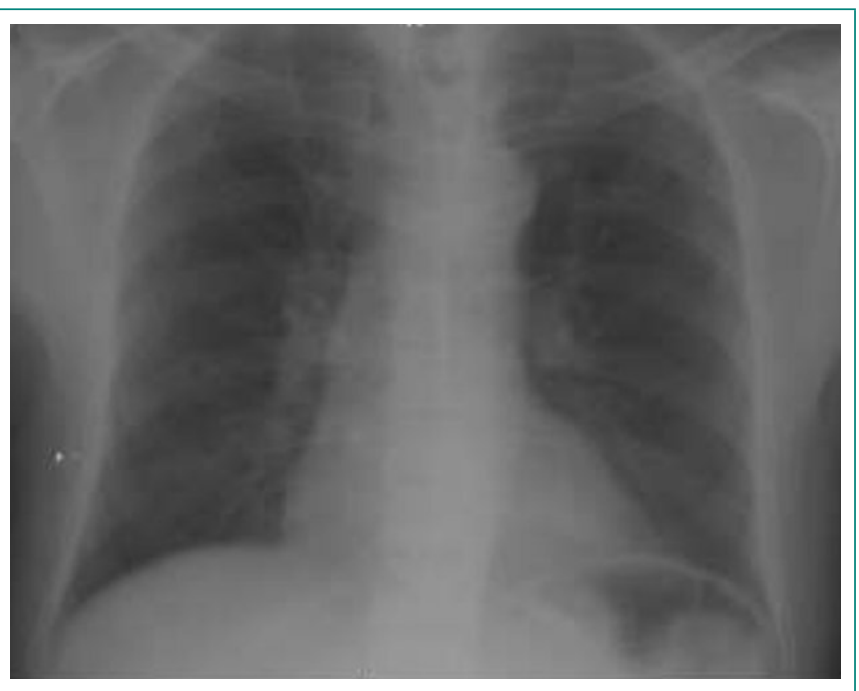

Figura 6. Radiografía de tórax: la expansibilidad pulmonar es simétrica. No hay lesiones consolidativas.

Se le solicitaron estudios para descartar la coinfección con sífilis, HIV, hepatitis B y C; los cuales fueron negativos. Adicionalmente, se inició terapia RIPE (Rifampicina, Isoniacida, Pirazinamida y Etambutol) ajustado al peso y función renal.

Posteriormente, el paciente evolucionó de forma favorable completando su esquema de tratamiento durante 9 meses.

\section{Diagnóstico}

Tuberculosis hepática aislada en paciente inmunodeprimido por terapia renal sustitutiva.

\section{Discusión y conclusión}

En la presentación extrapulmonar de la TB, la forma hepática se ha considerado como una forma rara ${ }^{4}$. En cuanto a su fisiopatología, se considera que la mayoría de los casos de tuberculosis con afectación hepática son secundarias a una TB miliar que, por vía hematógena, migran los bacilos a la región hepática a través de la arteria hepática o la vena porta. En estos casos, el tracto respiratorio y el sistema gastrointestinal son los primeros en recibir la infección y, posteriormente, se produce la afectación hepática 5 .

La primera descripción se remonta al año 1958 realizada por Bristowe como TB hepática aislada o primaria ${ }^{5}$; posteriormente, Levine la clasifico en 5 formas, que incluyen: (i) TB miliar, (ii) TB pulmonar primaria con afectación hepática, (iii) TB primaria de hígado, (iv) tuberculoma, y (v) colangitis tuberculosa ${ }^{6}$. El presente caso corresponde a la tercera variante.

La TB hepática primaria es extremadamente rara, incluso en países donde la TB es un problema de salud pública ${ }^{2}$, con una prevalencia la cual apenas alcanza el 0,0015\% de todas las presentaciones de TB, como se muestra en un estudio realizado en Brunéi ${ }^{7}$, con una presentación clínica consistente en pérdida de peso (64\%), dolor abdominal (57,1\%), fiebre (50\%), ictericia $(42,3 \%)$ y distensión abdominal (14,3\%). Asimismo, la hepatomegalia y esplenomegalia se encuentran en $95 \%$ y $18-55 \%$ de los casos, respectivamente $e^{8}$

Los hallazgos bioquímicos pueden incluir inversión de la relación albuminaglobulina, elevación de AST, ALT y fosfatasa alcalina9. Esta presentación de síntomas y hallazgos analíticos tan inespecíficos llevan a que la TB hepática primaria sea confundida comúnmente con otras entidades más prevalentes, tales como: hepatocarcinoma, absceso hepático piógeno y metástasis hepáticas de canceres primarios. Teniendo en cuenta lo anterior, se considera que hay un retraso en el diagnóstico de aproximadamente 15 días (rango, 1-420 días) ${ }^{8}$ llevando a una morbilidad y mortalidad significativas?.

En cuanto al diagnóstico por imagen, los hallazgos radiológicos presentan una especificad baja; el ultrasonido y la tomografía computarizada evidencian granulomas asociado a necrosis caseosa y, en ocasiones, fibrosis y calcificaciones; también se pueden observar imágenes correspondientes a lesiones hipoecoicas e hipodensas respectivamente, de forma similar a las de nuestro paciente. Sin embargo, en otros casos se han encontrado lesiones hiperdensas e hiperecoicas; por ende, el diagnóstico definitivo depende de la demostración del agente etiológico por reacción en cadena de polimerasa (PCR) o en aspirado 5 . En este caso el diagnóstico se hizo mediante tinción de Ziehl-Neelsen sobre la muestra biopsica.

En la actualidad el tratamiento continúa siendo objeto de controversia debido a la presencia de tejido fibroso de los abscesos y el tamaño pueden impedir la penetración de los antibiótico; con la consecuente necesidad de realizar drenaje percutáneo en combinación con perfusión de fármacos antituberculsos a través de cáteter ${ }^{5}$. Sin embargo, en nuestro medio no se dispone de este servicio, por esta razón se indicó tratamiento antituberculoso oral durante 9 meses.

Este es un caso raro de TB hepática primaria, imposible distinguir inicialmente de metástasis hepáticas de un cáncer de origen desconocido a través de las técnicas de imagen. Además, los síntomas como dolor abdominal e hiporexia no eran específicos, además estaba afebril. Estos datos junto a la normalidad en la radiografía de tórax y baciloscopias seriadas de esputo dificultaron el diagnóstico. Este es el cuarto caso publicado en la literatura anglosajona de tuberculosis hepática aislada en pacientes que se encuentran en diálisis ${ }^{10-12}$. 


\section{Bibliografía}

1. Dheda K, Barry CE 3rd, Maartens G. Tuberculosis. Lancet. 2016; 387(10024): 1211-26. doi: 10.1016/S0140-6736(15)00151-8.

2. Kayar Y, Ekinci I, Turkdogan FT, Atay M, Soytas RB, Kayar NB. A rare case of isolated macronodular hepatic tuberculosis (Tuberculous) in an immunocompetent patient. J Pak Med Assoc. 2015; 65(11): 1235-6.

3. Bandyopadhyay S, Maity PK. Hepatobiliary tuberculosis. J Assoc Physicians India. 2013; 61(6): 404-7.

4. Hasan R, Kumar S, Mathew M, Kadavigere R. Isolated hepatosplenic tuberculosis: a rare presentation. BMJ Case Rep. 2015; 2015: bcr2015211065. doi: 10.1136/bcr-2015-211065.

5. Baveja C, Gumma V, Chaudhary M, Jha H. Primary tubercular liver abscess in an immunocompetent adult: a case report. J Med Case Rep. 2009; 3: 78. doi 10.1186/1752-1947-3-78.

6. Levine C. Primary macronodular hepatic tuberculosis: US and CT appearances. Gastrointest Radiol. 1990; 15(4): 307-9. doi: 10.1007/BF01888805.
7. Chong VH. Hepatobiliary tuberculosis: a review of presentations and outcomes. South Med J. 2008; 101(4): 356-61. doi: 10.1097/ SMJ.0b013e318164ddbb.

8. Desai CS, Josh AG, Abraham P, Desai DC, Deshpande RB, Bhaduri A et al. Hepatic tuberculosis in absence of disseminated abdominal tuberculosis. Ann Hepatol. 2006; 5(1): 41-3.

9. Chien RN, Lin PY, Liaw YF. Hepatic tuberculosis: comparison of miliary and local form. Infection. 1995; 23(1): 5-8. doi: 10.1007/BF01710049.

10. Chen HC, Chao YC, Shyu RY, Hsieh TY. Isolated tuberculous liver abscesses with multiple hyperechoic masses on ultrasound: a case report and review of the literature. Liver Int. 2003; 23(5): 346-50. doi: 10.1034/j.14783231.2003.00861.x.

11. Kriki P, Thodis E, Deftereos S, Panagoutsos S, Theodoridis M, Kantartzi K et al. A tumor-like manifestation of extrapulmonary tuberculosis in a hemodialysis patient. Clin Nephrol. 2009; 71(6): 714-8. doi: 10.5414/cnp71714.

12. Hung YM, Huang NC, Wang JS, Wann SR. Isolated hepatic tuberculosis mimicking liver tumors in a dialysis patient. Hemodial Int. 2015; 19(2): 344-6. doi: 10.1111/hdi.12205 